\title{
Development of Co-processed Paracetamol with Hydroxypropyl Methylcellulose (HPMC) and Maltodextrin by Wet Granulation Process
}

\author{
Ortega Leticia, Gómez Martín, Daniela Rodríguez \\ Department of Biological Systems, Metropolitan Autonomous University-Campus Xochimilco (UAM-X), Mexico City, Mexico
}

Email address:

lortegaa@correo.xoc.uam.mx (O. Leticia)

To cite this article:

Ortega Leticia, Gómez Martín, Daniela Rodríguez. Development of Co-processed Paracetamol with Hydroxypropyl Methylcellulose (HPMC) and Maltodextrin by Wet Granulation Process. Journal of Drug Design and Medicinal Chemistry. Vol. 5, No. 2, 2019 , pp. 26-32. doi: $10.11648 /$ j.jddmc.20190502.12

Received: July 5, 2019; Accepted: July 22, 2019; Published: August 7, 2019

\begin{abstract}
The purpose of this work is to improve one of the important physicochemical properties of an active pharmaceutical ingredient (API), as is the water solubility of paracetamol. To improve the physicochemical properties of this API, two pharmaceutical excipients such as the HPMC and maltodextrin were used, which help to improve the solubility, and this helps to the manufacturing process of a pharmaceutical product. Different granule formulations were manufactured by applying a matrix design of experiment where the wet granulation process was performed, combining Paracetamol with the excipients to obtain a uniform particle size and subsequently evaluate the properties of interest. The solubility was evaluated using a method (Mexican pharmacopoeia - FEUM) based on UV / VIS method, performing the calibration curve only for the API to evaluate the granule and calculate the percentage of solubility of these. Favorable results were obtained for two of the seven granule formulations manufactured, the mixture of granule F and G: $25 \mathrm{~g}$ of paracetamol, $1.5 ; 1.75 \mathrm{~g}$ of HPMC and 23.5 g; 23.25 of the maltodextrin has a solubility of $104.17 \%$ and $101.48 \%$ of the $G$, which shows that the process by wet granulation can improve its solubility. This type of co-processed granule also fulfills the function of masking the bitter taste of paracetamol in one oral pharmaceutical form, as in the case of a syrup and it could be an advantage in the market. The flavor was evaluated by a panel of 20 people and the taste of the syrups that were prepared with the granule with better solubility was compared with the syrups containing only the API dissolved. It is shown that the granules F have improvement in the solubility of paracetamol and can mask the unpleasant (bitter) taste of the active ingredient.
\end{abstract}

Keywords: Paracetamol, HPMC, Maltodextrin, Granulation

\section{Introduction}

Paracetamol (Figure 1a) is an analgesic and antipyretic drug widely used by the Mexican population for the control of mild or moderate pain. It is known that the pharmacological effect depends on the physicochemical characteristics of the active principle and one of the most important is the solubility; in the FEUM (Pharmacopoeia of the United Mexican States) specifies that Paracetamol is easily soluble in alcohol and methanol; soluble in acetone, hot water and in $1 \mathrm{~N}$ sodium hydroxide solution; almost insoluble in chloroform and diethyl ether [1].

To obtain better bioavailability there is an interest in improving the solubility of paracetamol in water at 25 (room temperature) and 37 degrees Celsius. The objective in this work was preparing granule by the wet granulation process.

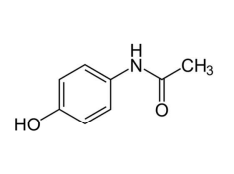

a)

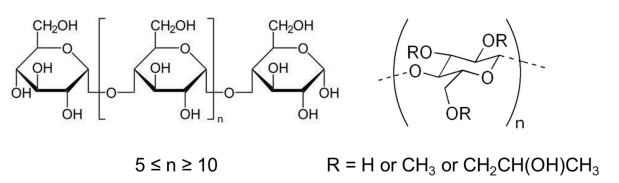

b)

c)
Figure 1. Chemical structure a) Paracetamol, b) maltodextrin and c) hydroxypropyl methylcellulose (HPMC).

The characteristics of the solute conditioning the solubility are the size and shape of the particle, the amorphous or crystalline state, the polymorphism, the density, the melting 
temperature (melting point) and the porosity rate among other properties of the solid phase. Also, the solubility is conditioned by the solvent and its wetting capacity, in particular the surface tension between the water and the solute (paracetamol) is relevant [2].

The solubility of drugs is one of the factors considered in the studies of pre-formulation of pharmaceutical forms, since depending on the value of solubility in water it will be determined if it is feasible to formulate pharmaceutical products such as tablets, capsules, suspensions or any other pharmaceutical form [2].

All pharmaceutical active ingredients (API's), regardless of the route by which they are administered, must be at least slightly soluble in water so that they can show absorption and have therapeutic efficacy, based on the principle that the drug must be soluble in water. the liquids present in the place where the absorption occurs [3].

There are currently numerous strategies aimed at modifying the solubility of the active ingredients used in the manufacture of medicines, which do not require changes in the chemical structure, but which can modify their bioavailability [4].

There are different articles that seek to improve the solubility of Paracetamol, Salima Varona and collaborators use different mixtures of excipients such as PVP and $\mathrm{CO} 2$ using high pressure equipment to increase the solubility [5].

Considering that the solubility of Paracetamol is one of the most important properties, experiments are carried out mixing different concentrations of solvents such as ethanol, acetone, and toluene [6].

Another advantage that can be obtained from the granulation of the APIs is the modification of the flavor. Flavor has always been a critical characteristic for the development of liquid pharmaceutical forms. However, currently, due to the expansion of solid dosage forms prior to administration or in the mouth, taste has become a critical parameter also in solid forms. Not only is it a matter of masking an unpleasant taste, but also a pleasant taste must be added to the formulation that increases patient acceptance [7].

In this investigation the process of functionality of the active ingredient was developed, which represents the modification of the physical structure of the particles of Paracetamol by introducing raw materials that are compatible with it and are able to improve the physicochemical properties of interest. It is proposed that by decreasing the particle size and dispersing Paracetamol in another excipient to promote solubility, so the strategy of wet granulation will help to improve the taste of the paracetamol contained in a pharmaceutical form. The granulation is prepared by mixing Paracetamol with HPMC (Hydroxypropyl methylcellulose) and Maltodextrin.

HPMC and Maltodextrin are the pharmaceutical excipients that can be included in the manufacture of a drug product [8], these substances help in the processing of the system during its manufacture, protect, improve stability, bioavailability or patient's acceptability, help in the identification of the product and improvement of any other general safety attribute, effectiveness or delivery of the medication during storage or use [8].

Maltodextrin is a mixture of saccharide polymers of Dglucose obtained by acid or enzymatic hydrolysis of foodgrade starches, followed by filtration, concentration, and drying of the product (Figure 1b) [9].

It is a white or almost white powder or granule, slightly hygroscopic, easily soluble in water and poorly soluble in $96 \%$ ethanol [9]. It is an inert additive favoring the recovery of the product since they act as coadjutants of drying, also for obtaining a higher yield [10].

Although it is a glucose polymer, it is not sweetener agent. It is used as a diluting excipient in capsules and tablets, as a tablet binder (both by direct compression and by wet granulation), as a coating agent in aqueous tablet solution, and as a viscosity-increasing agent (for example, to prevent crystallization in oral liquid preparations) [9].

HPMC (Hydroxypropyl methylcellulose) is a polymer derived from partially O-methylated and O- (2hydroxypropyl) propylated cellulose (Figure 1c) [11].

This excipient is characterized as a white or yellowishwhite powder or granule; it is hygroscopic, practically insoluble in hot water, acetone, anhydrous ethanol and toluene and soluble in cold water [11].

HPMC is used in the preparation of oral and topical pharmaceutical forms, presenting properties and uses like methylcellulose, although its mucilage has greater clarity and less non-dispersible fibers, being preferred in the preparation of ophthalmic solutions. Being non-ionic, HPMC is compatible with metallic salts and ionic organic compounds. It is stable in solutions with $\mathrm{pH}=3-11$. It is the only one, together with hydroxypropyl cellulose, which resists up to $100 \%$ alcohol. In oral preparations, it is used as a binder in wet or dry granulation, enteric coating, depending on the degree of viscosity, and as a matrix of controlled release tablets, using high viscosity Hypromellose [11].

The HPMC dose is related to its function, $2 \%$ as gelling agent (between 2 and 5\% as a binder in granulation ${ }^{7}$, between $2-10 \%$ to enteric coating [11].

\section{Methodology}

\subsection{Materials and Equipment Used}

Paracetamol (acetaminophen) Lot Y152381, Maltodextrin from Makymat Lot AR1187, HPMC (Hydroxypropyl methylcellulose) Lot 6075665, in sugar. Reagents: Hydrochloric Acid, Lot 9535-02, Iodine Lot M-32476, Sodium Hydroxide Lot 40254, Nitric Acid Lot 9621-02, Silver Nitrate, Methanol Lot 9070-03, Equipment: Sieves of $1.41 \mathrm{~mm}$ and $0.297 \mathrm{~mm}$, analytical scale SHIMADZU, laboratory scale, laboratory stove BG, pH-meter (potentiometer) 320 Corning, powder flowmeter, thermo scale OHAUS, blender (mixer), density meter, UV-vis Perkin Elmer. 


\subsection{Experiment Design}

Seven different granule formulations are proposed, with the composition obtained from the results of a previous work focused on preparation of granule (Table 1). This table shows the quantities of the raw materials to obtain $50 \mathrm{~g}$ of each granule formula (batch) approximately, the elaboration of the different batches of granule was in random order.

Table 1. Content of excipients and for the preparation of granules.

\begin{tabular}{lllllll}
\hline \multirow{2}{*}{ Granules } & \multicolumn{2}{l}{ Paracetamol } & \multicolumn{2}{l}{ HPMC } & \multicolumn{2}{l}{ Maltodextrin } \\
\cline { 2 - 7 } & $\mathbf{\%}$ & $\mathbf{g}$ & $\mathbf{\%}$ & $\mathbf{g}$ & $\mathbf{\%}$ & $\mathbf{g}$ \\
\hline A & 50 & 25 & 2.5 & 1.25 & 47.5 & 23.75 \\
B & 50 & 25 & 2.0 & 1 & 48 & 24 \\
C & 50 & 25 & 1.5 & 0.75 & 48.5 & 24.25 \\
D & 50 & 25 & 1 & 0.5 & 49 & 24.5 \\
E & 50 & 25 & 0.5 & 0.25 & 49.5 & 24.75 \\
F & 50 & 25 & 3.0 & 1.5 & 47 & 23.5 \\
G & 50 & 25 & 3.5 & 1.75 & 46.5 & 23.25 \\
\hline
\end{tabular}

The physicochemical properties evaluated to the raw materials and to the granule manufactured were bulk and compacted density, identity tests, flow velocity and angle of repose.

\subsection{Process Development}

Granulation process (for each granulate a duplicate of the same formulation was made)

1. The raw materials (Paracetamol, HPMC and Maltodextrin) are weighed on the analytical scale

2. Once the raw materials are weighted, they are placed in the mixer, the mixer is closed properly, the raw materials are mixed for 5 minutes at $30 \mathrm{rpm}$

3. At the end of the mixing time, the wet powder is placed on the tray without spreading through it, it remains in mountain form.

4. It begins to moisten the powder with the help of an atomizer containing distilled water, this way of adding helps us to make the homogeneous distribution of water.

5. To know if the moistening of the powder is adequate, touch and take a little amount and closing the hand with pressure during 5 seconds, when opening the hand we have to observe if the moistened powder is broken or a kind of molded mass is maintained, if not continue to wet with water slightly.

6. If a molded mass is maintained it already has a good humidification, the amount of water used is recorded and it is passed through a sieve with a mesh of 1.41 $\mathrm{mm}$, which will give us a good particle size when is dry.

7. When the granule is obtained passed through the mesh, it is extended a little by the tray and the it is placed in the stove, in which the temperature need to be $60^{\circ} \mathrm{C}$.

8. When the temperature of $60^{\circ} \mathrm{C}$ is reached, the granules will stay in the stove for 2 hours

9. After that time the humidity percentage in the thermo scale (loss by drying) is taken. The percentage of humidity to obtain is from $1 \%$ to $1.5 \%$. If you do not have the expected humidity, the granule is maintained in the stove, all of this will depend on the humidity obtained.

10. Pass through a sieve with a mesh of $0.297 \mathrm{~mm}$, at the end of this step, the powder will be stored in a bag previously labeled.

\section{Results}

Evaluation of the properties of paracetamol granules

The content of paracetamol was determined to the granule obtained and syrups were prepared to submit them to a flavor panel to assess and qualify their flavor, in addition to the physical tests.

Table 2. Flow properties of raw materials and granules.

\begin{tabular}{lllll}
\hline Raw materials or granulates & Carr Index & Hausner Index & Evaluation of flow properties & \% of humidity \\
\hline Paracetamol & 37.64 & 1.6 & Very poor and extremely bad & \\
HPMC & 38.3 & 1.62 & Extremely bad & 1.02 \\
Maltodextrin & 20.59 & 1.25 & Acceptable & 1.28 \\
Granulate A-1 & 24.98 & 1.33 & Poor & 1.10 \\
Granulate B-1 & 27.25 & 1.37 & Poor & 1.34 \\
Granulate C-1 & 24.98 & 1.33 & Poor & 1.36 \\
Granulate D-1 & 25.71 & 1.34 & Poor & 1.48 \\
Granulate E-1 & 25.23 & 1.33 & Poor & 1.34 \\
Granulate F-1 & 25.63 & 1.34 & Poor & 1.38 \\
Granulate G-1 & 25.67 & 1.34 & Poor & 1.30 \\
Granulate A-2 & 28.43 & 1.39 & Poor & 1.20 \\
Granulate B-2 & 31.64 & 1.46 & Poor and very poor & 1.12 \\
Granulate C-2 & 31.89 & 1.46 & Poor & 1.28 \\
Granulate D-2 & 30.35 & Poor & 1.10 \\
Granulate E-2 & 31.26 & Poor & 1.02 \\
Granulate F-2 & 30.16 & 1.43 & Poor and very poor & 1.15 \\
Granulate G-2 & 33.33 & Poor and very poor & 1.17 \\
Granulate F-2' & 31 & Too poor & \\
Granulate G-2' & 33.5 & 1.45 & 1.5 & \\
\hline
\end{tabular}




\section{Evaluation of paracetamol}

The spectrophotometric evaluation for the content of Paracetamol in the granules was carried out based on method established in the Mexican pharmacopoeia (FEUM, 2019. EVALUATION, MGA 0361).

$120 \mathrm{mg}$ of paracetamol standard was dissolved in $10 \mathrm{~mL}$ of methanol and it was brought to a $500 \mathrm{~mL}$ capacity with water. From this solution, the calibration curve is constructed by measuring the absorbance at a wave length of $243 \mathrm{~nm}$.

Preparation of the sample, an amount of solid equivalent to 120 $\mathrm{mg}$ of paracetamol (API or granules) is weighed, it is perfectly dissolved in $10 \mathrm{ml}$ of methanol and it is brought to a total volume of $500 \mathrm{ml}$ with water. A dilution of the sample 1 in 20 is prepared with five replicates, filtered and the absorbance is determined at $243 \mathrm{~nm}$, using water for the absorbance adjustment, and calculating the percentage of paracetamol contained in the sample, considering the amount of solid and the dilution made.

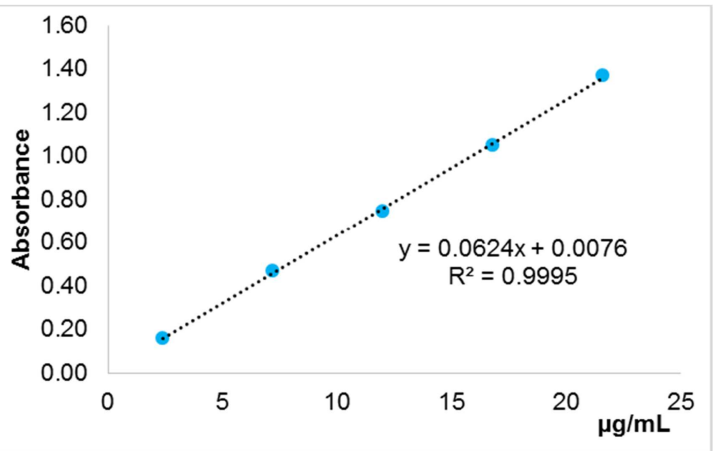

Figure 2. Spectrophotometric calibration curve of Paracetamol in water, at $243 \mathrm{~nm}$.
Figure 2 represents the spectrophotometric calibration curve of Paracetamol at $243 \mathrm{~nm}$, in water, using methanol as a cosolvent. From this calibration, the amount of API released from the different granules can be evaluated. The evaluation is done fifth times, considering that upon dissolution of $100 \%$ of the active principle, the solution will have a concentration of $12 \mu \mathrm{g} / \mathrm{mL}$ (Table 3 )

The evaluation of the amount of Paracetamol dissolved from the granules is obtained by converting the absorbances into concentration using the equation of the UV-VIS calibration curve. Considering the theoretical concentration of active ingredient as $100 \%$, the percentage of dissolved Paracetamol (Table 3) is calculated. It is observed that when changing batch there is an increase in the soluble amount of Paracetamol, it is related to the formulation and to the optimization of the evaluation procedure.

Table 3 presents the percentage of dissolved paracetamol from the granules, the absorbances are compared with the calibration curve of Paracetamol by the UV-VIS method, and according to the expected theoretical value of active ingredient, the percentage of soluble Paracetamol is calculated. The increase in the soluble amount of Paracetamol is due to the formulation and the ability to develop the evaluation. As Paracetamol is hepatotoxic it is important to improve its solubility having better bioavailability but reducing de amount dosed. The gastrointestinal absorption is conditioned by: the solubility (which limits the concentration of a drug that can be found in solution and the speed at which the molecules are dissolved).

Table 3. Percentage of dissolved paracetamol from the granules, evaluated by spectrophotometry.

\begin{tabular}{llll}
\hline Granulate & Theoretical concentration $\boldsymbol{\mu g} / \mathbf{m L}$ & Batch 1\% & Batch 2\% \\
\hline A & 12 & 31,3 & 71,5 \\
B & 12 & 31,9 & 72,3 \\
C & 12 & 33,5 & 74,7 \\
D & 12 & 49,7 & 76,4 \\
E & 12 & 46,7 & 85,3 \\
F & 12 & 61,3 & 100,9 \\
G & 12 & 70,4 & 100,3 \\
\hline
\end{tabular}

Syrup preparation procedure

Simple syrup $500 \mathrm{~mL}$

1. $445 \mathrm{~g}$ of refined sugar are weighed in a beaker

2. In another beaker with a capacity of 1 liter, add $250 \mathrm{~mL}$ of distilled water, place a magnet to stir the water and gradually add the $445 \mathrm{~g}$ of refined sugar.

3. Maintain the agitation until the sugar dissolves completely, for this also the temperature of the solution rises slightly without letting it reach boiling point.

Taste tests

1. Six samples will be made which will contain $20 \mathrm{~mL}$ each of simple syrup.

2. Each sample will be labeled with a different percentage of $0.25 \%, 0.5 \%, 0.75 \%, 1.0 \%, 1.25 \%$ and $1.5 \%$ (Scheme 5), this percentage represents the amount of flavor added to the samples. Note: $0.25 \%$ equals 0.3
$\mathrm{mL}$.

3. With the samples ready, 10 people are chosen at random and of different ages (panel), to test the samples and thereby determine a percentage of acceptable taste.

Paracetamol Syrup

1. $1.6 \mathrm{~g}$ of Paracetamol is weighed.

2. In a beaker, $14.3 \mathrm{~mL}$ of propylene glycol are added to dissolve the paracetamol, for this purpose magnetic stirring is used.

3. When the paracetamol is dissolved, take it up to $50 \mathrm{~mL}$ with simple syrup and leave it in agitation to obtain a homogeneous solution.

4. $0.6 \mathrm{~mL}$ (equivalent to $0.5 \%$ ) of Tutti-frutti flavoring is added.

Syrup with the granules

1. $3.2 \mathrm{~g}$ of Paracetamol is weighed. 
2. In a beaker, $14.3 \mathrm{~mL}$ of propylene glycol are added to dissolve the Paracetamol granules, for which purpose magnetic stirring is used.

3. When Paracetamol granules are dissolved it is taken up to $50 \mathrm{~mL}$ with simple syrup and maintain in agitation to have a homogeneous solution.

4. $0.6 \mathrm{~mL}$ (equivalent to $0.5 \%$ ) of Tutti-frutti flavor is added.

A survey was conducted to evaluate the taste of syrups made with Paracetamol or granules with Paracetamol, the original taste of Paracetamol is unpleasant for people. The results of 20 people for flavor evaluation are shown in Table 4 , these results can be combined with the taste of other syrups (Table 5), containing co-processed products manufactured in another project. For evaluate the taste there are different techniques, both in vivo and in vitro, widely developed and described by Garcia Espada et al. This study used the evaluation gustatory-sensorial in humans (Taste trials) since it is the method most used today [7].

This test is carried out in healthy individuals that after retaining in the oral cavity for a certain time the pharmaceutical form to be evaluated, their sensory experience must be evaluated. For evaluate temporal profiles of taste masking, the operation is repeated at different times.

In order to standardize subjectivity at the time of classifying the flavor experienced or used different scales such as numerical, symbols such as,,$-+++\ldots$ visual analogue, descriptive. In this work we used a format that is a mixture of symbols that describe a result and is quantified according to the number of participants in the panel [7]

Table 4. Evaluation of the taste masking of the paracetamol contained in granulates, 20 participants are considered in the panel.

\begin{tabular}{llll}
\hline Jarabe & Unpleasant & Tolerable & Pleasant \\
\hline API with flavoring & 10 & 8 & 2 \\
Granulate F without flavoring & 6 & 11 & 3 \\
Granulate G without flavoring & 9 & 4 & 7 \\
API with flavoring & 1 & 12 & 7 \\
Granulate F with flavoring & 2 & 7 & 11 \\
Granulate G with flavoring & 5 & 10 & 5 \\
\hline
\end{tabular}

Table 5. Evaluation of the taste masking of the Paracetamol contained in coprocessed.

\begin{tabular}{llll}
\hline Syrup & Unpleasant & Tolerable & Pleasant \\
\hline Co-processed A without flavoring & 3 & 2 & 0 \\
Co-procesado A with flavoring & 1 & 3 & 1 \\
Co-procesado B without flavoring & 3 & 1 & 1 \\
Co-procesado B with flavoring & 0 & 2 & 3 \\
\hline
\end{tabular}

\section{Discussion}

Based on the literature and these results it is verified that the parameters established for each excipient used (maltodextrin and HPMC) and the API (Paracetamol) meet the identity test established by FEUM [12]. Maltodextrin and HPMC were added to the dosage within the limits to comply appropriately in safety and are included in the delivery systems of drug products to aid in processing or manufacturing, to protect, support and improve stability, bioavailability or acceptability of patient, to support the identification of the product when is required, to improve other safety attributes and effectiveness of the dosage form, during storage and during its use [10]. In this project, maltodextrin and HPMC excipients and the wet granulation method, are proposed to improve physicochemical properties and flavor.

In Table 1 presents the formulas with quantities of excipients used for preparation of seven different granule formulation and percentage of each component (Paracetamol, maltodextrin and HPMC) shown per granule formulation. Based on the results of the evaluation for the physical properties of the seven granule formulations (Table 2), the granules identified with improved pharmaceutical properties with respect to the objectives of this work, are the granule formulations F and G. Also, it can see that for some granule formulations the properties as flow velocity and angle of repose are not favorable although these properties are not part of the primary objective to improve.

The pharmaceutical preformulation studies show various problems related to the physicochemical properties of the active ingredient (paracetamol). Thus one can also detect whether the drug has unpleasant organoleptic characteristics such as odor or taste, which can cause the rejection by the patient, which could be associated with the failure of the prescription and possible therapeutic failure [4]. In this case the paracetamol is characterized by a low solubility and by its bad taste, so the granule formulations manufactured are evaluated for solubility of Paracetamol using the UV-VIS spectrophotometry when this powder is dissolved, and thus determine what granule formula has a major percentage of solubility of the active ingredient. It is noted as the granule $\mathrm{F}$ having a content of $25 \mathrm{~g}$ of Paracetamol, $1.5 \mathrm{~g}$ of HPMC and $23.5 \mathrm{~g}$ of maltodextrin, dissolves Acetaminophen (paracetamol) achieving $102.80 \%$ (considering the experimental error); and granules $\mathrm{G}$ with $25 \mathrm{~g}$ of Paracetamol, $1.75 \mathrm{~g}$ of HPMC and $23.25 \mathrm{~g}$ of Maltodextrin has $102.29 \%$ of Acetaminophen dissolved in the batch 2. By repeating the manufacture of the granules $F$ and $G$, in the granule $F$ the solubility achieved was $104.17 \%$ and for the $G$ the solubility was $101.48 \%$. With these results it can be established that wet granulation method to obtain formulations and using $3.0 \%$ and $3.5 \%$ of HPMC with maltodextrin as diluent, generates an improvement in the solubility of paracetamol defining HPMC as the functional excipient in this objective.

Another important factor that was evaluated is the taste of Paracetamol, as it was already explained in the methodology. A flavor panel was made to the syrups containing only paracetamol in simple solution and containing granules form in solution and the granules $F$ and $G$ that presented a better solubility, for tasting the flavor panel, the way to qualify was modified to make it simpler according to Espada García and Chiappetta, using the survey of Figure 3 applied to 20 volunteers chosen at random from 20 to 55 years old, they do not know what syrup was manufactured using Paracetamol 
simple or any of the syrups with granules selected, and if the syrup contained flavoring or not [13]

The results are presented in Table 4 . The results show that the syrup containing the API without flavoring is unpleasant for 10 people and with flavoring it is tolerable for 12 people; the syrup with granules $\mathrm{F}$ without flavoring is tolerable for 11 people and with flavoring it becomes pleasant for 11 people; the syrup with granulated $G$ is unpleasant for 9 people and with flavoring it becomes pleasant for 10 people. The results of this taste panel allow to verify that the granule formulation in the syrup also helps to mask the taste of Paracetamol, mainly using the granules $\mathrm{F}$.

In a previous research work was developed a coprocessing with HPMC and Maltodextrin with Paracetamol by spray drying, to improve its properties. This co-processing of these substances also defines the formation of excipients with superior physicochemical properties compared with the values with simple physical mixtures. It is a method to obtain a product with the desired optimized physicochemical properties, especially for solid dosage forms [14].

\section{UNIVERSIDAD AUTÓNOMA METROPOLITANA}

\begin{tabular}{ll} 
& Taste Panel \\
Name: & Age: \\
Occupation: & F__ \\
\hline
\end{tabular}

Instructions: test each syrup and select the emoticon that represents your taste perception.
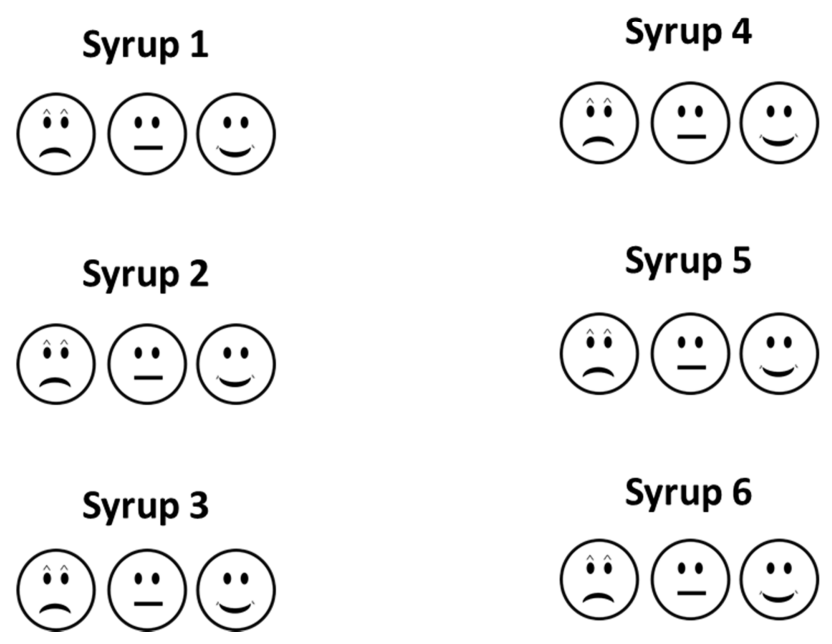

Figure 3. Survey for the taste panel. The sad face means unpleasant, the second face represents half tolerable and the happy face is associated with a pleasant taste.

With the co-processed mix obtained by spray dry method that showed an improvement in the solubility, its flavor was evaluated with a flavor panel with 5 people. When manufacturing the syrups with the paracetamol co-processed, it was detected that it does not dissolve all material entirety, but that it can be used in improving solid formulations. The results obtained from the taste panel for two co-processed are shown in Table 5, determining that the syrup with co- processed A without flavoring is unpleasant for three people and with flavoring becomes tolerable for 3 people; the syrup with co-processed B without flavoring is unpleasant for 3 people and becomes pleasant for 3 people. With the results obtained with the co-processed paracetamol we can determine that the taste is masked with the co-processed B this co-processed contains $25 \mathrm{~g}$ of Paracetamol, $1 \mathrm{~g}$ of HPMC and $24 \mathrm{~g}$ of Maltodextrin.

Comparing the results of masking the taste of paracetamol in a syrup, containing the granule formulations with the coprocessed method, a better strategy is the manufacture of paracetamol granules by wet granulation way, for the liquid pharmaceutical forms. The low solubility is a disadvantage for the paracetamol co-processed and this impacts the solubility leaving particulate material in the syrup related with the bitter taste. On the other hand, paracetamol granulates $\mathrm{F}$ and $\mathrm{G}$ dissolve completely, taking into account that appearance is an important factor for the acceptance by the patient of this pharmaceutical form.

The results presented in this work correlate with that reported by Afrasiabi, which showed that the aqueous solubility of paracetamol in the presence of polyvinylpyrrolidone (PVP) increased. The solubility at $25^{\circ} \mathrm{C}$ increased from $14.3 \mathrm{mg} / \mathrm{mL}$ in the presence of PVP, to 19.7 $\mathrm{mg} / \mathrm{mL}$ in the presence of $4 \% \mathrm{p} / \mathrm{v}$ of PVP, and to $26.7 \mathrm{mg} / \mathrm{mL}$ in the presence of $8 \% \mathrm{p} / \mathrm{v}$ PVP [15].

It is proposed to continue with this research in order to improve the results obtained from physicochemical properties, solubility and taste adding some functional test (flowability). Other excipients proportions with the best results will be obtained and new excipients reported in the literature will be incorporated for better results. It will choose those that are available in the market, that are safety, economical and non-toxic.

\section{Conclusion}

These results show that wet granulation strategy, is an effective method to obtain a semi finished products of Paracetamol with excipients HPMC and maltodextrin improving one of the physicochemical property most important for the drug formulation such as solubility and dissolution in water of Paracetamol.

An improvement in the solubility of paracetamol is important at an industrial level since it will allow a lower content of paracetamol to achieve the concentration and dose in the formulation, with a lower cost of the product as a consequence.

It was achieved masking the bitter taste of the active ingredient present in medicine with paracetamol in this project; the taste was evaluated in a simple syrup and was demonstrated that taste is improved for people, in some case these results were improved by using a more structured formulation.

To carry out more experiments and new pharmaceutical forms allow confirm or improve the results obtained for paracetamol in this work. 


\section{Acknowledgements}

Laboratory of excipients No. 112 in the Metropolitan Autonomous University Campus Xochimilco.

Collaboration of Makymat SA of CV.

Alfredo Salazar G., R\&D Director of Laboratorios Liomont SA de CV.

\section{References}

[1] FEUM, F. d. (2018). Paracetamol (12th ed., Vol. 1). of Mexico: Secretary of Health.

[2] Isabel González Álvarez, M. Á. (2015). Biopharmaceutical methodologies in the development of medicines (08-06-15 ed.). Miguel Hernández University of Elche.

[3] Herazo, EA (2012). Physicochemical study of the solubility of acetaminophen in polyethylene glycol $400+$ water mixtures. Thesis, Bogotá.

[4] Domínguez-Gil, AM (2011). Royal National Academy of Pharmacy; Advances in pharmaceutical technology. Retrieved March 29, 2018 from http://www.analesranf.com/index.php/mono/article/view/532/ 550.

[5] Varona S, Fernández J, Rossmann M and Braeuer A (2013). Solubility of Paracetamol and Polyvinylpyrrolidone in Mixtures of Carbon Dioxide, Ethanol, and Acetone at Elevated Pressures. American Chemical Society. 58, 10541061.

[6] Ruether F and Sadowski G (2009). Modeling the Solubility of Pharmaceuticals in Pure Solvents and Solvent Mixtures for Drug Process Design. Wiley InterScience. DOI 10.1002/ jps. 21725 .

[7] Espada García JI, Fernandes Tavares DF, Fraguas Sánchez AI,
Aparicio Blanco J, Martín Sabroso C, Torres Suárez AI (2015). Masking of flavors in solid oral dosage forms. Annals of the Royal Academy of Pharmacy (1697-4298), 116-128.

[8] IPEC, TI (2006). The Join IPEC- PQG Good Manufacturing Practices Guide for pharmaceutical excipients.

[9] Acofarma (nd) Tokens of technical information. Maltodextrin Retrieved March 29, 2018 from http://www.acofarma.com/admin/uploads/descarga/659665aa65d75536417fb925977904912902b92d2316/main/files/ Maltodextrina.pdf.

[10] López, OM (2006). Influence of the use of additives on the performance of the spray drying process of aqueous extract of Calendula officinalis L. Cuban Journal of Medicinal Plants.

[11] Acofarma (nd) Hydroxypropyl Methyl Cellulose. Retrieved August 31, 2017 from http://www.acofarma.com/admin/uploads/descarga/437347a1 ee7b1d50cddacc89770a35df329dd1da0fdc/main/files/Hi droxipropil_metil_celulosa.pdf.

[12] Villafuerte, L. (2011). The excipients and their functionality in solid pharmaceutical products. Scielo, 42 (1), 18-36.

[13] Chiappetta DA, Rodríguez Llimós AC, Witte NL, Széliga ME, Niselman V and Bregni C (2004). Enmascaramiento de Sabor de Paracetamol Utilizando Microencapsulación. Acta Farm. Bonaerense 23 (3): 292-6.

[14] Minakshi Marwaha, DS (2010). Coprocessing of Excipients: A Review on Excipient Development For Improved Tabletting Performance. International Journal of Applied Pharmaceutics, 2 (3), 41-47.

[15] Afrasiabi Garekani H, Sadeghi F and Ghazi A (2003). Increasing the Aqueous Solubility of Acetaminophen in the Presence of Polyvinylpyrrolidone and Investigation of the Mechanisms Involved, Drug Development and Industrial Pharmacy, 29: 2, 173-179, DOI: 10.1081/DDC-120016725. 